\title{
An Adaptive QSE-reduced Nuclear Reaction Network for Silicon Burning
}

\author{
Suzanne Parete-Koon* ${ }^{*+}$ \\ University of Tennessee \\ E-mail: snp@utk.edu \\ W.R. Hix \\ Oak Ridge National Laboratory \\ E-mail: raph@ornl.gov
}

\section{F.-K. Thielemann}

University of Basel

E-mail: f-k.thielemann@unibas.ch

The nuclei of the "iron peak" are formed in massive stars shortly before core collapse and during their supernova outbursts as well as during thermonuclear supernovae. Complete and incomplete silicon burning during these events are responsible for the production of a wide range of nuclei with atomic mass numbers from 28 to 64 . Because of the large number of nuclei involved, accurate modeling of silicon burning is computationally expensive. However, examination of the physics of silicon burning has revealed that the nuclear evolution is dominated by large groups of nuclei in mutual equilibrium. We present an improvement on our hybrid equilibrium-network scheme which takes advantage of this quasi-equilibrium in order to reduce the number of independent variables calculated. Because the size and membership of these groups vary as the temperature, density and electron faction change, achieving maximal efficiency requires dynamic adjustment of group number and membership. Toward this end, we are implementing a scheme beginning with 2 QSE groups at appropriately high temperature, then progressing through, 3 and 3* group stages (with successively more independent variables) as temperature declines. This combination allows accurate prediction of the nuclear abundance evolution, deleptonization and energy generation at a further reduced computational cost when compared to a conventional nuclear reaction network or our previous 3 fixed group QSE-reduced network. During silicon burning, the resultant QSE-reduced network is up to 20 times faster than the full network it replaces without significant loss of accuracy. These reductions in computational cost and the number of species evolved make QSE-reduced networks well suited for inclusion within hydrodynamic simulations, particularly in multi-dimensional applications.

10th Symposium on Nuclei in the Cosmos

July 27 - August 12008

Mackinac Island, Michigan, USA

\footnotetext{
${ }^{*}$ Speaker.

†This work has been supported by the National Science Foundation, by theDepartment of Energy’s Scientific Discovery through AdvancedComputing Programs, and by the Joint Institute for Heavy Ion Research at ORNL.
} 


\section{Introduction}

In Supernovae simulations nucleosynthesis calculations predict the thermonuclear energy generated by the nuclear reactions and predict the abundances of the species produced during the explosion. Due to great computational cost of modeling the hydrodynamics, modelers often neglect or crudely approximate the nucleosynthesis, during the hydrodynamics simulation and attempted to recapture the details of the nucleosynthesis later with post-processing. This work explores how the physics of chemical equilibrium can be used to make more accurate low computational cost nucleosynthesis calculation that could be included in situ in a hydrodynamics simulation.

Quasi-Statistical Equilibrium (QSE) forms when light particle capture reactions and their inverse photo-dissociations run in equilibrium within isolated groups of nuclei [1].

The number, size, and membership of these QSE groups vary with temperature, density, and electron fraction [2]. During explosive silicon burning, for temperatures in excess of $6 \mathrm{GK}$ the QSE groups merge to form a Global Nuclear Statistical Equilibrium (NSE).

A matrix calculation is at the heart of a nuclear reaction network. The speed of this calculation scales nonlinearly with the number of species in the network. A typical reaction network requires about 300 species and over 3000 reactions to model Silicon burning [3].

Only one variable representing the nucelar species must be followed for each QSE group in the network matrix calculation and reactions between group members do not require calculation of reaction rates. Therefore, QSE can increase the speed of the network by reducing the number of variables in the matrix calculation and the number of reactions that must be used.

\section{The Physics of Chemical Equilibrium}

The physics of chemical equilibrium follows from the chemical potential. For a group of nuclei in QSE, the chemical potential of any group member can be expressed in terms of the sum of the chemical potentials of the proton, neutron, and one focal nucleus chosen from the group members:

$$
\mu_{A Z}=\mu_{\text {focal }}+\left(Z_{A_{Z}}-Z_{\text {focal }}\right) \mu_{p}+\left(N_{A Z}-N_{\text {focal }}\right) \mu_{n}
$$

Here $\left(Z_{A Z}-Z_{\text {focal }}\right)$ and $\left(N_{A Z}-N_{\text {focal }}\right)$ are the number of protons and neutrons need to build the species ${ }^{A} Z$ from the focal nucleus. For a group of particles obeying Boltzmann statistics, the chemical potential of each species can be written

$$
\mu_{A_{Z}}=m_{A_{Z}} c^{2}+k_{\mathrm{B}} \ln \left[\frac{Y_{A_{Z}} \rho N_{a}}{G_{A_{Z}}}\left(\frac{2 \pi \hbar^{2}}{m_{u} k_{\mathrm{B}} T}\right)^{3 / 2}\right],
$$

Where $m_{A} Z$ is the mass of the species ${ }^{A} Z, c$ is the speed of light, $k_{\mathrm{B}}$ is Boltzmann's constant, $Y_{{ }^{A} Z}$, is the abundance of the species ${ }^{A} Z, G_{A} Z$ is the partition function, $\rho$ is the density and $T$ is the temperature.

Equation 2.2 can be substituted into equation 2.1 and solved to get an expression for the abundances of the ith element.

$$
Y_{\mathrm{QSE}, \text { focal }}\left({ }^{A} Z\right)=\frac{C\left({ }^{A} Z\right)}{C\left(Y_{\text {focal }}\right) Y_{p}^{Z-Z_{\text {focal }}} Y_{n}^{N-N_{\text {focal }}}},
$$


where we have defined

$$
C\left({ }^{A} Z\right)=\frac{G\left({ }^{A} Z\right)}{2^{A}}\left(\frac{\rho N_{A}}{\theta}\right)^{A-1} A^{\frac{3}{2}} \exp \left(\frac{B\left({ }^{A} Z\right)}{k_{\mathrm{B}} T}\right)
$$

and

$$
\theta=\left(\frac{m_{u} k_{\mathrm{B}} T}{2 \pi \hbar^{2}}\right)^{3 / 2}
$$

$A$ is the atomic number. $N$ is the neutron number. $B\left({ }^{A} Z\right)$ is binding energy of the nucleus ${ }^{A} Z . N_{A}$ is Avogadro's number. $Y_{p}$ and $Y_{n}$ are the abundances of free protons and neutrons and the integers $\left(N_{A Z}-N_{\text {focal }}\right)$ and $\left(Z_{A Z}-Z_{\text {focal }}\right)$ are the number of such nucleons needed to construct the nucleus ${ }^{A} Z$ from the focal nucleus. Thus to follow the evolution of every member of a QSE group through conditions of the changing temperature and density, one only needs to track the abundances of protons, neutrons, and one focal nucleus. For simplicity this expression was derived in the absence of electron screening of the nucleus.

\section{Testing the QSE Network}

Initial conditions of $5 \mathrm{GK}$ coupled with a peak density of $10^{9} \mathrm{~g} \mathrm{~cm}^{-3}$ lead to incomplete silicon burning. To approximate the evolution of the density and temperature during a supernova explosion, the widely used prescription introduced by Fowler and Hoyle [4] was employed. The expansion is assumed to occur on the hydrodynamic timescale. We followed a composition of nuclei as it cooled from $5 \mathrm{GK}$ to $3 \mathrm{GK}$, the temperature range where QSE assumptions are valid for explosive conditions.

Figure 1 shows the QSE group membership of the 2-group, 3-group, and 3*-group configurations for the largest set of nuclei. The 2-group configuration uses 57 independent variables consisting of the independent nuclei, the proton and neutron group abundances, and one group abundance for its large QSE group. The 3-group configuration follows the evolution with 82 independent variables. The $3{ }^{*}$-group network uses 93 independent variables, which is less than a third the number need for the full network.

The adaptive QSE network switched automatically between the 2-group, 3-group, and 3*group configurations during the run. Figure 2 shows how the network switched between the QSE group configuration for the different adaptive runs used to explore the trade-offs between speed and accuracy.

Table 1 shows that all QSE-reduced network configurations performed at least 10 times faster than the full network, making them good candidates for use in hydrodynamic simulations. The left panel of Figure 3 shows that the energy generation rate of all QSE-reduced network trials matches that of the full network well until the temperatures falls below 3.5 GK. The adaptive 1 and 2 runs that start in the 3-group, rather than the 2-group configuration, are slower than than the adaptive 3 and 4 runs, but match the energy generation rate of the full network better at early times. Given that the adaptive 1 and 2 runs are still almost 20 times fasters than the full network and that the bulk of the nucleosynthesis occurs in the early high temperatures, the trade-off of better accuracy for slower speed is justified. The right panel of Figure 3 shows the detailed abundance composition at the end of the simulation for the adaptive 1 and 2 runs and a trial that ran with fixed 3-group 

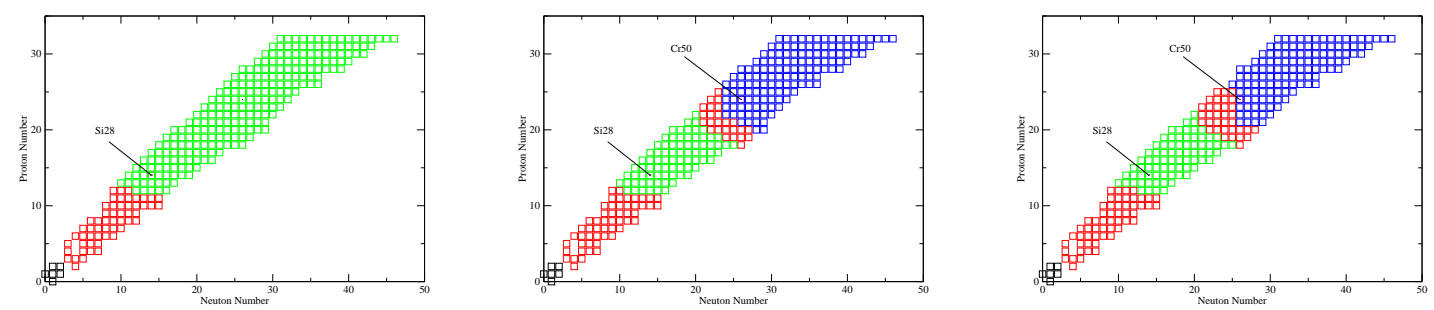

Figure 1 Silicon QSE-Group members are green, Iron-Peak QSE groups members are Blue, Light groups members are Black and independent species are Red.

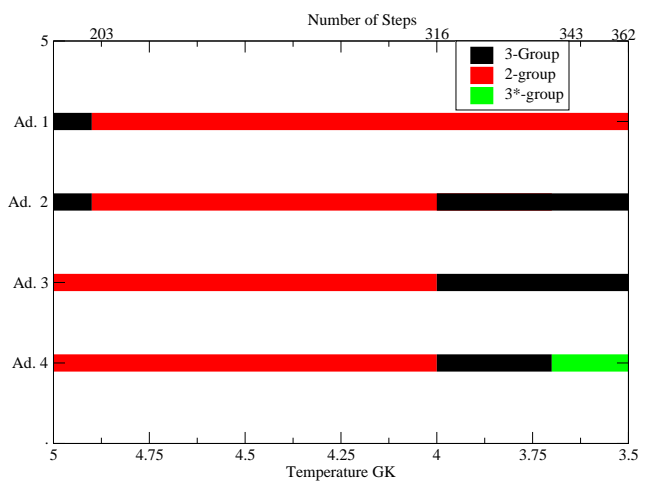

Figure 2 Shown are four variations for how the Adaptive QSE-reduced network can be tuned to switch between QSE group configurations during incomplete silicon burning.

Table 1. Network Speed for Incomplete Silicon Burning

\begin{tabular}{lcccccr}
\hline \hline Network & \multicolumn{3}{c}{ Core Duo } & \multicolumn{3}{c}{ Pentium4 } \\
& $\begin{array}{c}\text { cpu } \\
\text { time } \\
(\mathrm{s})\end{array}$ & $\begin{array}{c}\text { matrix } \\
\text { time } \\
(\%)\end{array}$ & $\begin{array}{c}\text { Iteration } \\
\text { count }\end{array}$ & $\begin{array}{c}\text { cpu } \\
\text { time } \\
(\mathrm{s})\end{array}$ & $\begin{array}{c}\text { matrix } \\
\text { time } \\
(\%)\end{array}$ & $\begin{array}{c}\text { Iteration } \\
\text { count }\end{array}$ \\
\multicolumn{7}{l}{ Vendor Supplied Solver } \\
3QSE & 1.07 & 45 & 1066 & 1.56 & 29 & 1065 \\
2QSE & 0.56 & 27 & 831 & 0.82 & 23 & 831 \\
Ad.1 & 0.83 & 30 & 991 & 1.12 & 28 & 950 \\
Ad.2 & 1.00 & 321 & 1035 & 1.46 & 28 & 985 \\
Ad.3 & 0.78 & 30 & 933 & 1.04 & 23 & 864 \\
Ad.4 & 0.95 & 30 & 978 & 1.25 & 22 & 921 \\
Full & 17.36 & 83 & 1155 & 18.48 & 82 & 1280 \\
\hline
\end{tabular}



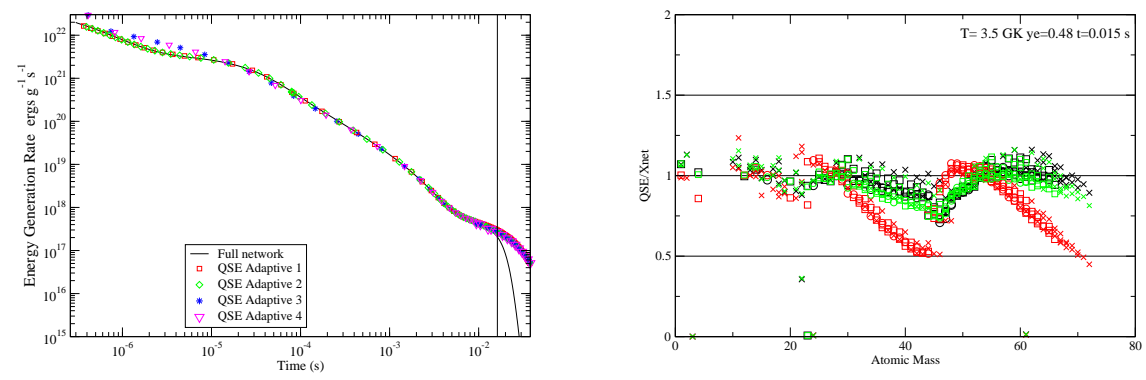

Figure 3 Shown on the left is a comparison of the energy generation rate of the full network and the QSE-reduced network for incomplete silicon burning. A vertical black line marks the time that corresponds to a temperature of $3.5 \mathrm{GK}$, after which the QSE assumption begins to fail. Shown on the right are the ratios between abundances calculated with the QSE-reduced network and those calculated by the full network for incomplete silicon burning. Black symbols indicate the fixed 3-group network, red indicate the Adaptive-1 run, and green indicate the Adaptive-2 run.

configuration for the whole simulation. The abundances from all runs except those of adaptive 2 run match those of the full network to 20 percent or better, but as table 1 shows, the adaptive runs deliver this accuracy at a greater speed than the fixed 3-group run.

By adapting the number and membership of the QSE groups, even better agreement with the abundances of the full network and/or further reduced computational cost can be achieved. The choice of how to apply this improvement will vary with the application. For many burning stages, where a single large QSE group is present, use of adaptivity allows the QSE network to run about 20 times faster than the full network. For more complex behavior, like that seen during alpharich burning, varying degrees of adaptivity can allow sufficient accuracy for the dominant species and energy generation with a large (15-20x shown in later trials) improvement in speed, or a high degree of accuracy even for trace species with a more modest improvement $(5 x)$ in speed. Decisions on how best to apply adaptivity to the QSE-reduced network within a hydrodynamic model also influence the treatment of advection and mixing. With dynamic QSE group allocation, the meaning of each group abundance can be different between adjoining hydrodynamic zones or mass elements, thus the hydrodynamic evolution can not take advantage of the reduction in the number of evolved abundances and must evolve the full abundance set. The use of QSE-group adaptivity should be cost-effective for models where the thermonuclear evolution dominates the computational cost, but fixed groups may be the proper choice for models which more closely balance the thermonuclear and hydrodynamic costs.

\section{References}

[1] Bodansky, D., Clayton, D.,\& Fowler, W. 1968, ApJS, 16, 299

[2] Hix, W. R., \& Thielemann, F.-K. 1996, ApJ, 460, 869

[3] Hix, W. R., \& Thielemann, F.-K. 1996, ApJ, 511, 862

[4] Fowler, W., \& Hoyle, F. 1964, ApJS, 9, 201 